\title{
Comment on 'A simple and powerful analytical model for MEMS piezoelectric multimorphs' by W.E. Booij, A.H. Vogl, D.T. Wang, F. Tyholdt, N.P. Østbø, H. Rader, K. Prume [J. Electroceram. 19, 387 (2007)]
}

\author{
X. H. Tan - A. K. Soh
}

Received: 2 July 2008 / Accepted: 21 April 2009 /Published online: 8 May 2009

(C) Springer Science + Business Media, LLC 2009

Keywords Piezoelectric multimorphs .

Electromechanical coupling $\cdot$ Lumped circuit model

In the paper, entitled 'A simple and powerful analytical model for MEMS piezoelectric multimorphs' [1], a lumped circuit model was proposed to describe MEMS device structures coupled with piezoelectric materials. In the said paper, the system was presented by equation (2) as follows:

$m \cdot \ddot{\kappa}+\gamma \cdot \dot{\kappa}+\frac{1}{C_{M}} \cdot \kappa-k_{m e 1} V=M_{e x t}(t)$

$\dot{q}-k_{m e 2} \dot{\kappa}-C_{p} \dot{V}-\frac{V}{R_{P}}=0$

The system was composed of a mechanical dynamic part and an electrical circuit part, which were coupled through two coupling coefficients $k_{m e 1}$ and $k_{m e 2}$. The derivation of the coupling coefficients was critical to the success of the modeling. The coefficient $k_{m e 1}$ was determined from the moment applied by the piezoelectric material onto the structure, which was meaningful. However, the derivation of $k_{m e 2}$ might not be reasonable.

In the said paper, $k_{m e 2}$ was determined by 'equating the total power delivered by an applied voltage in the electrical system to the power delivered to the mechanical system by the resulting moment'. However, the two powers are

X. H. Tan · A. K. Soh $(\bowtie)$

The University of Hong Kong,

Hong Kong, People's Republic of China

e-mail: aksoh@hku.hk generally not equal and they are related by a coupling coefficient. By the definition provided by Mason [2],

$k^{2}=($ stored mechanical energy/supplied electrical energy $)$

or

$k^{2}=($ stored electrical energy/supplied mechanical energy $)$

where $k$ is the electromechanical coupling coefficient, which is totally related to the material constants. For the existing piezoelectric materials, $k^{2} \neq 1$.

In fact, the definition of the electromechanical coupling coefficient clearly states that not all the energy input in one form (either mechanical or electrical) can be totally converted to another form (either electrical or mechanical). Thus, $k^{2}<1$. Therefore, the method of obtaining $k_{m e 2}$ in the said paper should be modified by including the coupling coefficient $k^{2}$. The inconvertible part of energy is associated with the difference in compliances of the short circuited and the open circuited conditions.

In the said paper, some discrepancies were found in the curve fitting, for which the authors provided some explanations. We feel that omission of the effect arising from the electromechanical coupling coefficient of the coupling system could also lead to such discrepancies.

\section{References}

1. W.E. Booij, A.H. Vogl, D.T. Wang, F. Tyholdt, N.P. Østbø, H. Ræder et al., J. Electroceram. 19, 387 (2007). doi:10.1007/ s10832-007-9034-5

2. T. Ikeda, Fundamentals of piezoelectricity (Oxford University, Oxford, 1996), pp. 5-30. 209-240 